\title{
Electrical Counting Characteristics of Several Equivolume Micro-organisms
}

\author{
BY W. A. CURBY, ELLEN M. SWANTON AND H. E. LIND \\ I.S.R. Sias Laboratories, Brookline 46, Massachusetts, U.S.A.
}

(Received 24, September 1962)

\begin{abstract}
SUMMARY
Several micro-organisms of similar initial size $\left(0 \cdot 25 \mu^{3}\right)$ were used to study the intra-species and inter-genus variations in the electronic counting characteristics and population distribution as functions of culture age. A. Coulter Counter Model A was used to count populations of Staphylococcus aureus SM, Escherichia coli (Sias), an $\boldsymbol{E}$. coli variant, $E$. freundii (8454), and an $E$. freundii variant. Initial inocula into brain heart infusion broth were from agar slopes. Cultures were incubated at $37^{\circ}$ for various growth periods from 4 to $24 \mathrm{hr}$.; centrifuged at $1500 \mathrm{~g}$ for $25 \mathrm{~min}$, and the deposit resuspended in $0.9 \%(\mathrm{w} / \mathrm{v}) \mathrm{NaCl}$ twice, then resuspended in $10 \mathrm{ml}$. saline, diluted $1 / 10^{4}$, shaken, and counted in the Coulter counter at maximum gain on aperture current settings (a.c.s.) 4, 5 and 6 with threshold settings from 5 to 100. Population distributions were made with a phase-contrast microscope in a Petroff-Hauser counting chamber. The numbers of aggregates containing $1,2,3,4$ and $>4$ organisms were recorded as percentage of total populations. Counts were established for all bacterial populations tested as well as for the $0 \cdot 81 \mu$ diameter latex sphere counting standard. With a technique based on the addition of random variables, true bacterial population counts could be formulated. Some of the actively dividing populations displayed characteristic sensitivities which jwere dependent in part on the aperture current setting. It is concluded that intra-species as well as inter-genus variations exist. These variations are the result of factors such as: the given organism, its age, distribution and growth rate at the time of count, dilution and counting media, aperture diameter and magnitude of the current field in the electronic counting system.
\end{abstract}

\section{INTRODUCTION}

The counting of bacteria in suspension by a method which gives immediate values for population size is desirable. The study of subtle and rapid changes in the growth of given bacterial populations has been limited. Preliminary reports of progress in the field of electronic counting of bacteria have appeared recently (Toennies, Iszard, Rogers \& Shockman, 1961; Truant, Brett \& Merckel, 1962). Our previous work on this problem has shown the necessity of working with populations of one organism by using basic microbiological techniques, and by referring frequently to established standards (Swanton, Curby \& Lind, 1962). As with many complex functions, certain general relationships exist which make possible the solution of the function under special conditions. The present paper is concerned with the rationale involved in attaining a practicable means of counting bacterial 
suspensions by using the Coulter Counter and to show the results obtained when applying this method to counting different micro-organisms of similar initial volume $\left(0 \cdot 25 \mu^{3}\right)$.

\section{METHODS}

Staphylococcus aureus SM, Escherichia coli Sias, an $E$. coli variant, $E$. freundii (8454), and an $E$. freundii variant were grown in brain heart infusion broth (supplied by Difco Laboratories Inc., Detroit, Michigan, U.S.A.) and followed for periods from $6 \mathrm{~min}$. to $48 \mathrm{hr}$. Pour plates were made for each culture at the time of inoculation and at $1 \mathrm{hr}$. intervals thereafter. Simultaneously, counts were taken on a Model A Coulter Counter, adjusted for maximum gain, from $0.5 \mathrm{ml}$. samples of the

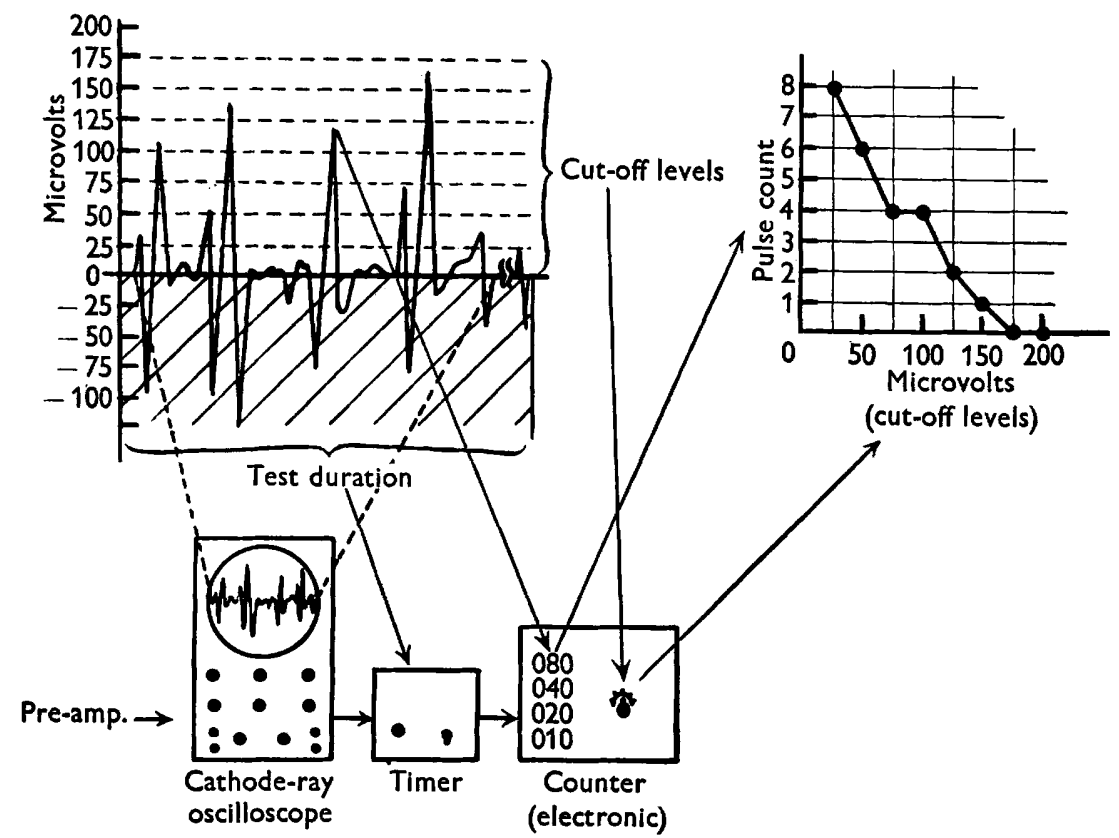

Fig. 1. Pictorial diagram of typical pulse counting equipment together with a graph insert (upper right) showing how the data are displayed in subsequent figures. For simplicity a linear rather than a semi-log or log-log relationship is depicted. The counter used in our experiments has a fixed volume rather than a fixed time interval control, but for ease of graphic explanation a fixed time unit is shown.

growing culture diluted in $0.9 \%(\mathrm{w} / \mathrm{v})$ sodium chloride solution. All counts were made through a $30 \mu$ diameter aperture of the apparatus at threshold settings from 5 to 100 for aperture current settings (a.c.s.) 4, 5 and 6. Population distributions and visual counts were made on samples introduced into a phase-illuminated Petroff-Hausser counting chamber. The electronic counting method used for this study utilized two volumes of a fluid several hundred times more conductive than the particles which were to be counted. The two volumes of fluid were continuous through a precision hole ( $30 \mu$ diameter) drilled in a thin, non-conductive wall. In each of the volumes a non-reactive platinum electrode was submerged, and a fixed current electric circuit was completed between them through the fluid suspension. 
A known volume $(0.05 \mathrm{ml}$.) of fluid with its suspended particles was caused to flow through the aperture. As each particle passed through the orifice, there was a transient change in resistance. A voltage increase (current $\times$ resistance) occurred each time a particle passed through the aperture, the amplitude of the voltage pulse generated being proportional to the aperture-filling properties of the particle (mainly size and shape). These voltage pulses were amplified and passed through a circuit which controlled the minimum voltage that was registered on a connected pulse-counter. Figure 1 shows the counting circuitry; at the upper right a method of graphically indicating the results obtained as the threshold cut-off value is increased is shown. The voltage pulses generated as the particles were pulled through the aperture were amplified and displayed on the cathode-ray tube. Pulses of similar polarity were passed into a counting circuit through a unit which controlled the test interval as a function of time or, as in our experiments, of volume. The effect of varying the threshold cut-off value may be followed (Fig. 1) in the expanded view of the image on the cathode-ray tube superimposed on the pulse height grid (upper left part of figure). It will be noted that 8 pulses in the test duration were $25 \mu \mathrm{V}$. in amplitude or greater. This was displayed as a pulse count of 8 at a threshold cut-off value of $\mathbf{2 5}$ on the graph in the upper right part of the figure. There were 4 pulses having amplitudes of $75 \mu \mathrm{V}$. or greater; in fact, the amplitude of these pulses was greater than $100 \mu \mathrm{V}$. Thus for both cut-off values the pulse count was the same. This flat portion of the graph will be referred to as a plateau, and the portion to the right of it as the tail-off of the curve. The curve at the $75 \mu \mathrm{V}$. threshold value made a positive flexure, while at the $100 \mu \mathrm{V}$. value it flexed negatively. Families of pulse count versus threshold curves were generated for each of our micro-organisms; one curve was noted for each $1 \mathrm{hr}$. interval in the growth period.

An analysis of the particle distribution producing a curve of the form described above is shown in Fig. 2. A complete treatment of the analysis of the addition of two randomly presented functions upon which this analytic method is based will be the subject of a subsequent publication. The dark line on the graph in Fig. $2 a$ is the resultant of two random (stochastic) variable curves indicated in dotted lines (Dixon \& Massey, 1957). The straight line with the negative slope would be generated if the particles in the fluid volume counted were randomly distributed as to rate and order of presentation, size, shape, and some factors of lesser importance. When all particles are uniform, but again presented randomly with respect to rate and order, the curve with the wide plateau is generated. The horizontal position of the plateau on the resultant curve is determined by the effective size of the particles. Using a $30 \mu$ diameter aperture under the aforementioned test conditions the tail-off of the particle count versus threshold setting curve for $0.81 \mu$ diam. latex spheres occurred at a threshold setting of 6 for an a.c.s. of 5, and at a threshold setting of 9 for an a.c.s. of 6 . Under the same conditions, the plateaux of the curves generated by $2 \cdot 85 \mu$ diam. latex spheres extended through threshold settings of 100 for a.c.s.'s of both 5 and 6 with a tail-off which began at 95 for a.c.s. 4. The maximum threshold setting seen under test conditions at which the tail-off began for bacteria studied was 90 for a.c.s. 6 . It was therefore possible to follow the growth of the organisms studied through their entire size increase in the growth cycle, and to estimate that the $30 \mu$ diameter aperture under test 
conditions described here will allow for the analysis of particles having space-filling properties equivalent to spherical volumes ranging from 0.25 to $12 \cdot 00 \mu^{3}$. The width and the vertical position was also altered by factors which were affected by the frequency of the pulses as well as the pulse amplitude. It should be pointed out that these factors limited the use of the analytical technique to be discussed to pulse input rates of about $6 \times 10^{3}$ pulses/sec. or less. Pulses generated at frequencies greater than this tended to superimpose in time and increase the apparent amplitude of the total pulse pattern. This theorefical limit was in accord with the findings of Brecher et al. (1962), who reported an increase in pulse counts at $8 \times 10^{3}$ erythrocytes/sec. especially at higher threshold values. The particles ordinarily found as

Particle counting characteristics, based mainly on size and shape distribution

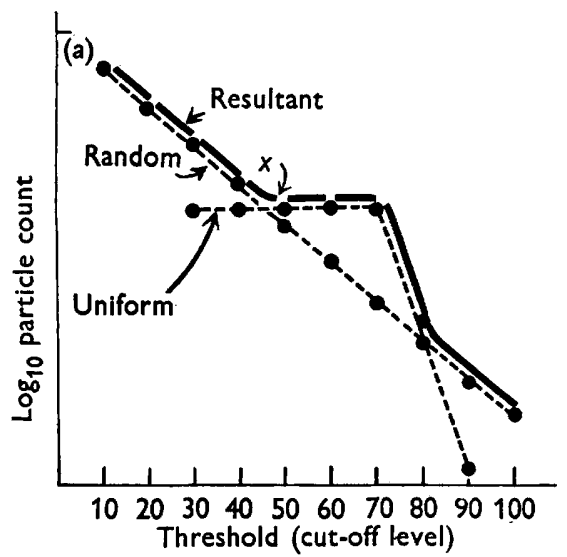

Growing bacterial population showing three time periods

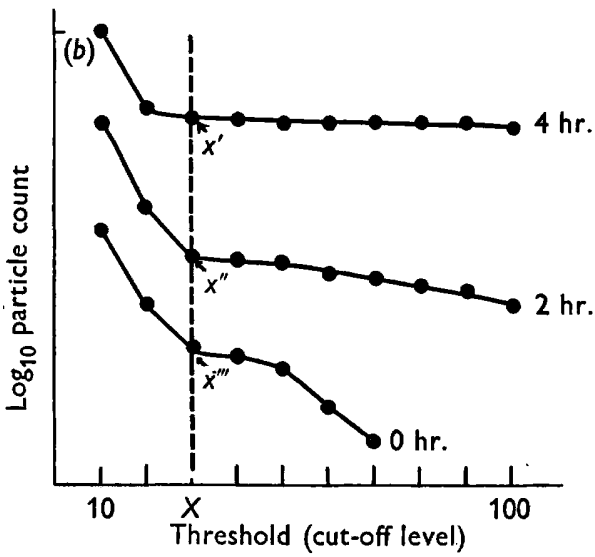

Fig. 2. (a). The resultant of the superimposition of populations of randomly and uniformly sized particle populations. Point $x$ shows the threshold level at which the count in a growing micro-organism population should be most reliable. (b). The typical growth curves generated from a growing bacterial population. In practice the cut-off level $(X)$ which best approximates point $x$ in graph $a$ will be used. Thus, particle counts for 0,2 and $4 \mathrm{hr}$. will be taken as points $x^{\prime}, x^{\prime \prime}$ and $x^{\prime \prime \prime}$ respectively.

impurities in suspension media used for counting were randomly distributed with respect to size and to some extent shape. The presence of these particles did not affect the total count of particles of uniform size analysed by the method described in this paper, provided their contribution did not cause the total pulse count/sec. to exceed the $6 \times 10^{3}$ limit.

The bacterial populations grown in pure cultures showed counting characteristics which followed those indicated in Fig. $2 b$. A reliable particle count could be made when enough uniform particles were present to cause a positive flexure in the curves. The mean percentage standard deviation for all counts within a pulse population range of $1.5 \times 10^{2}$ to $3.0 \times 10^{3}$ pulses/sec., and based on random sampling of the variance over a 6-month period under conditions recommended in this paper was found to be $\mathbf{1} .945$ within a minimum of 0.314 and a maximum of 6.565 . An electrical count having a variance smaller than that found for its associated standard plate count was possible if one took as a point of count the pulse count for the threshold 
value at the base of the first positive flexure on any of the curves; point $x$ in Fig. $2 a$, and points $x^{\prime}, x^{\prime \prime}, x^{\prime \prime \prime}$ in the curves in Fig. $2 b$. In mixed population analysis any subsequent positive flexures must also be considered. For all curves in a family generated from any micro-organism population, this threshold ( $X$ in Fig. $2 b$ ) can be taken as constant. To demonstrate the capabilities of this analytical procedure, no correction factors were used on any of the data presented.

\section{RESULTS}

Figures 3-7 show comparisons of growth characteristics, some population distributions for two cultures of Escherichia coli, two of E. freundii and one of Staphylococcus aureus; and the effect of the magnitude of current flow through the counting orifice (aperture current) on the reproducibility of the electrical count. Changing the aperture current setting (a.c.s.) control on the counter was observed to have an effect on the recorded particle count at times in the growth cycle of some of the organisms studied (Swanton et al. 1962). It can be demonstrated that this effect

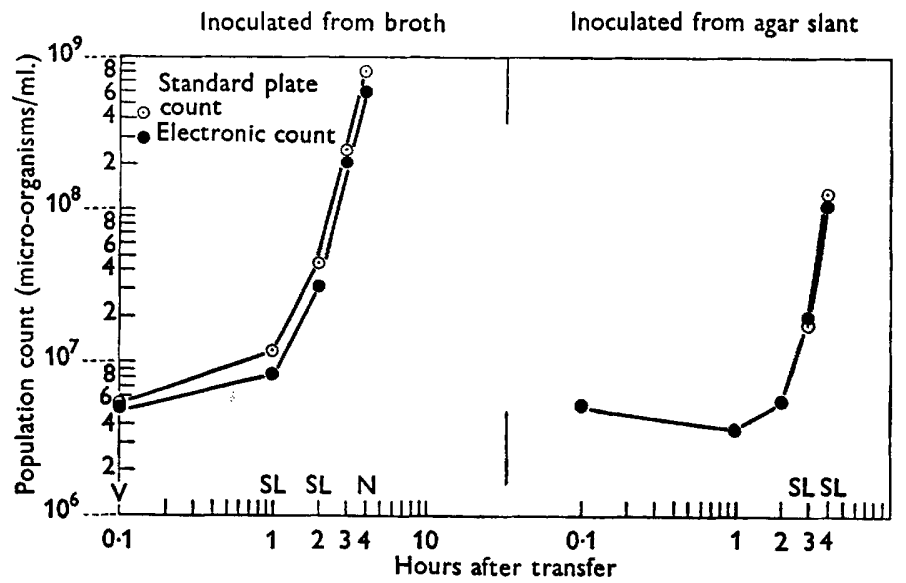

Fig. 3. Comparison of plate counts and electronic counts obtained from populations of Escherichia coli Sias. The notations V, SL and N above the abscissa indicate times at which the organisms were found to be, very, slightly, or not detectably sensitive to the current field in the counting media.

is associated with a general sensitivity of the micro-organisms to a variation in the magnitude of some electrical field property in the counting chamber. Until more definite information concerning this phenomenon can be obtained, we will refer to it as an electromotive force (E.M.F.) sensitivity. Figure 3 shows an $E$. coli and compares populations initiated from two inoculation sources. The degree of E.M.F. sensitivity, as measured by the failure of plateaux of curves in the graphs of particle count versus threshold setting to superimpose for aperture current settings of 4, 5 , and 6 will be seen just above the abscissa: V for very sensitive; SL for slightly sensitive; $\mathbf{N}$ for no detectable E.M.F. sensitivity. The filled circles represent the population counts made within $1 \mathrm{~min}$. after the electronic count began, while the open circles indicate the average values for plate counts read $24 \mathrm{hr}$. later. The maximum growth rate for this organism was 2.05 divisions $/ \mathrm{hr}$. when inoculated from broth and $2 \cdot 02$ divisions/hr. when inoculated from an agar slope. The $E$. coli 
variant results shown in Fig. 4 were from extended periods of study. PetroffHausser counts were made at the points in time indicated by the triangles. Population distributions given in percentage of singlets $(\mathrm{S})$, doublets $(\mathrm{D})$, triplets $(\mathrm{T})$, quadruplets $(\mathrm{Q})$, and greater than quadruplets $(>\mathrm{Q})$, appear in legends beside

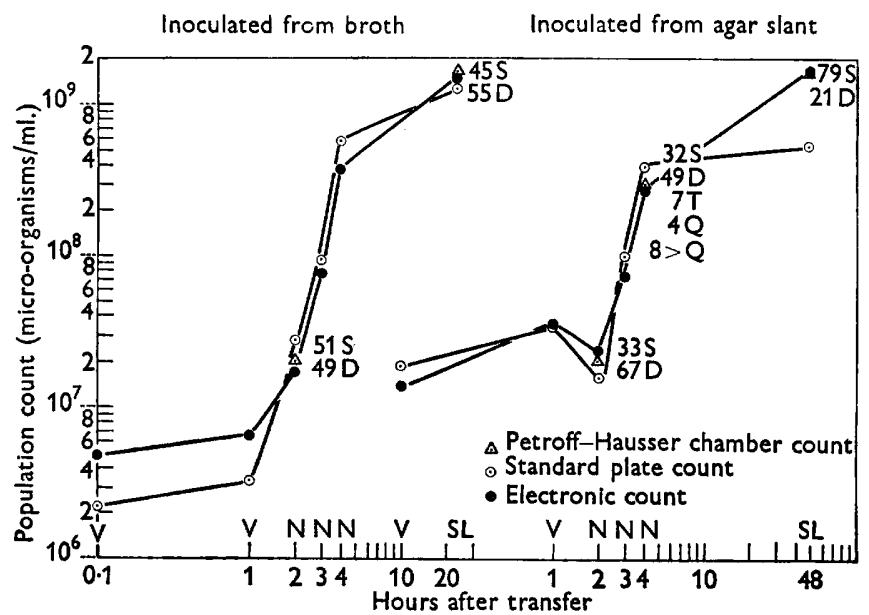

Fig. 4. Comparison of plate counts, electronic counts and Petroff-Hausser chamber counts obtained from populations of an Escherichia coli variant. The notations V, SL, and N above the abscissa indicate times at which the organisms were found to be: very, slightly and not detectably sensitive to the current field in the counting medium. The numbers beside the triangles indicate the percentage of singlets (S), doublets (D), triplets (T), and the quadruplets $(\mathbf{Q})$, and greater than quadruplets $(>\mathbf{Q})$ making up the population at the time of count.

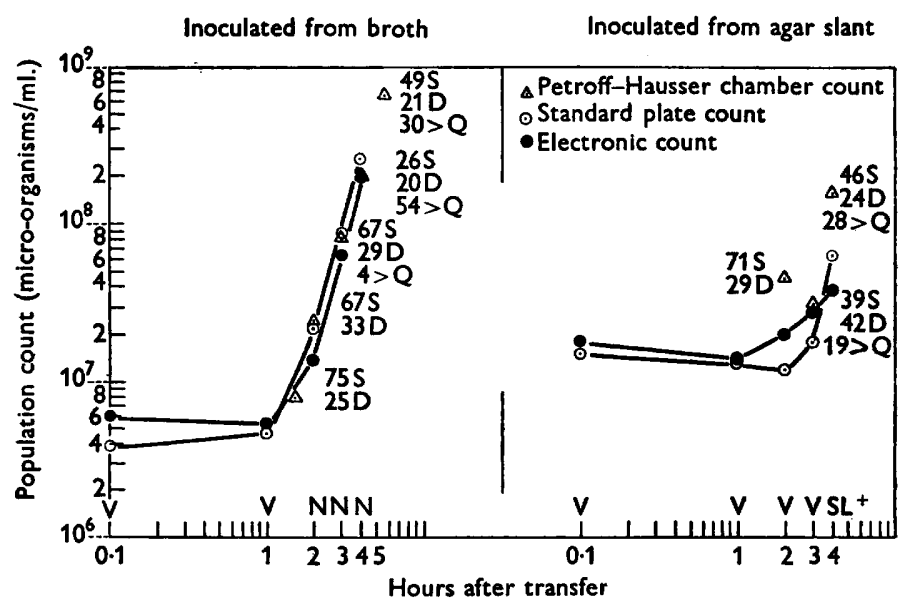

Fig. 5. Comparison of plate counts, electronic counts, and Petroff-Hausser counts obtained from populations of Escherichia freundii 8454. The notations $\mathrm{V}, \mathrm{SL}$, and $\mathrm{N}$ above the abscissa indicate the times during population growth at which the organisms were found to be: very, slightly, and not detectably sensitive to the current field in the counting medium. The numbers beside the triangles indicate the percentage of singlets (S), doublets (D), triplets (T), quadruplets (Q), and greater than quadruplets $(>\mathbf{Q})$ making up the population at the time of count. 
each triangle. At the $48 \mathrm{hr}$ interval for the culture grown from agar, the plate count was lower than the electrical count and the Petroff-Hausser count. This was probably because some dead organisms had accumulated which would not have been differentiated by methods based on physical counting techniques. This

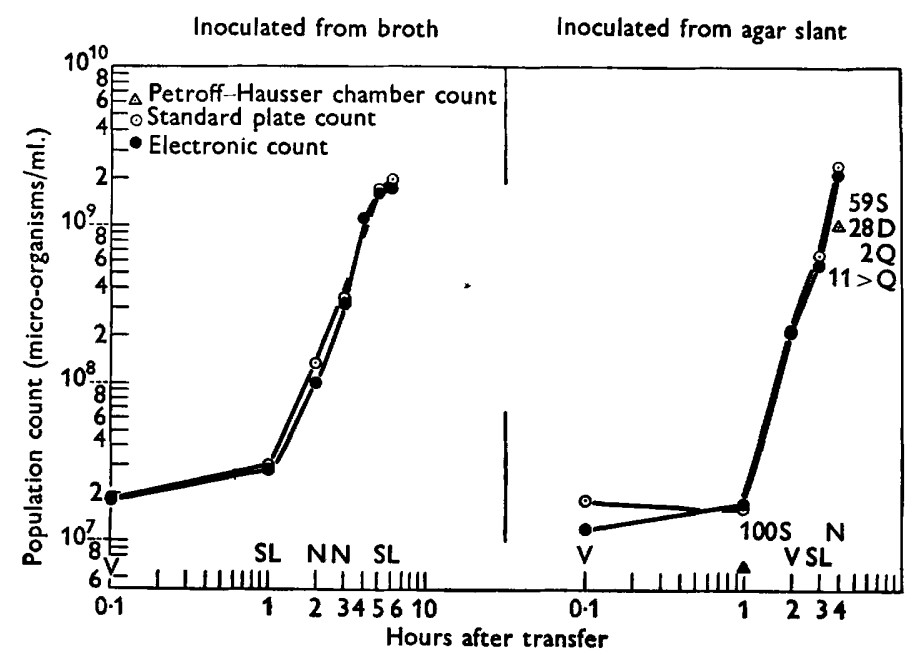

Fig. 6. Comparison of plate counts, electronic counts, and Petroff-Hausser counts obtained from populations of an Escherichia freundii variant. The notations V, SL, and N above the abscissa indicate the times during population growth at which the organisms were found to be : very, slightly, and not detectably sensitive to the current field in the counting medium. The numbers beside the triangles indicate the percentage of singlets (S), doublets (D), triplets (T), quadruplets (Q), and greater than quadruplets $(>Q)$ making up the population at the time of count.

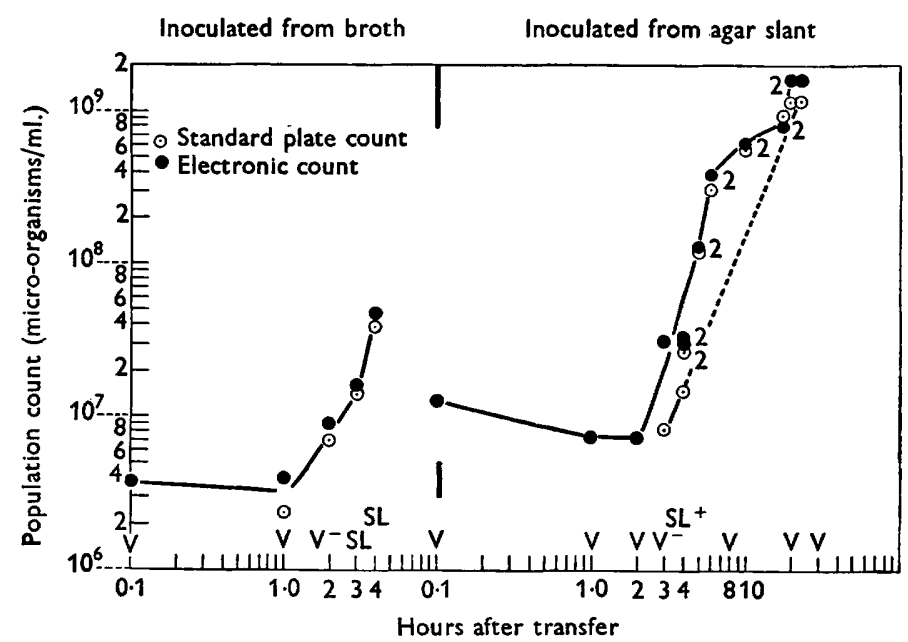

Fig. 7. Comparison of plate counts and electronic counts obtained from populations of Staphylococcus aureus SM. The points having the notation 2 in the graph of the microorganism grown from agar are plotted from a culture of the same stock two months after the other agar slope data were obtained. The notations V and SL indicate the times at which the cells were found to be very and slightly sensitive to the current field in the counting medium. 
difference can be seen to be slight in the $24 \mathrm{hr}$. sample from the culture grown from broth; the maximum growth rate was 2.00 divisions/hr. for the population started from broth and $\mathbf{2} \cdot \mathbf{1 7}$ divisions/hr. for the population initiated from an agar slope. $E$. freundii 8454 (Fig. 5) resembled $E$. coli, although it was also like $S$. aureus in its E.M.F. sensitivity. The plate counts may be related to the E.M.F. sensitivity. If the culture was highly sensitive, the plate count was consistently lower than the simultaneous electrical count. When no E.M.F. sensitivity was demonstrated the reverse occurred. The action of the population initiated from the agar slope was not clear. Subsequent studies may clarify the reasons for this behaviour. The maximum growth rate for the broth source culture for this bacterium was $1 \cdot 80$ divisions $/ \mathrm{hr}$. The maximum growth rate for the agar source culture was $1 \cdot 14$ divisions $/ \mathrm{hr}$. The population distributions indicated considerable clumping at times during growth. Because of the shape of these organisms and properties of the clumps, this did not affect the counting reliability. The $E$. freundii variant in Fig. 6 exhibited a sharp drop in E.M.F. sensitivity for the culture inoculated from broth. The sensitivity began to return at $6 \mathrm{hr}$. as the growth rate diminished from a maximum of 1.88 divisions/hr. The Petroff-Hausser counts taken at the 1 and $4 \mathrm{hr}$. test periods for the population started from an agar slope show the same growth rate $(2 \cdot 46$ divisions/ hr.) as that calculated from the other counting methods; however, the counts are lower. Further study will be necessary to explain or dismiss this as a recurrent phenomenon for this organism. S. aureus SM seen in Fig. 7 displayed continuously high E.M.F. sensitivity and again the electrical counts were higher than their plate count mates. The growth rate for these populations attained a maximum of $1 \cdot 22$ divisions/hr. for the population started from broth and 1.46 divisions/hr. for the populations inoculated from agar slopes. Two cultures inoculated from agar slopes were plotted together in this figure. The 0 and $4 \mathrm{hr}$. interval and the $24 \mathrm{hr}$. point were plotted from one culture; the 4 and $20 \mathrm{hr}$. interval from a culture started from the same stock 2 months later. The results indicated constant growth characteristics for this organism.

\section{DISCUSSION}

Several facts were apparent from the results. Staphylococcus aureus and one Escherichia freundii showed E.M.F. sensitivity throughout their growth period. All the micro-organisms studied exhibited the greatest E.M.F. sensitivity before the rapid growth phase. E.M.F. sensitivity had some effect on the electronic versus plate count. This sensitivity held regardless of polarity of the electrodes, thus showing that this phenomenon was not related to a charge on the organisms, but, rather, was determined by an active process within the organisms just before cell division. All of the Escherichia populations studied displayed similar growth characteristics. Population distribution did not seem to have an effect on the electrical count; however, the tendency to clog the aperture increased as the percentage of greater than quadruple units became larger. All Escherichia species examined showed normal frequency distribution for singlets and doublets except at the time of maximum growth. $S$. aureus did not follow the normal distribution. As would be expected in theory, by using the analytical method here discussed, the electronic count correlation with the standard plate count remained high. Because of the shift in population distribution during portions of the growth cycles 
of some of the bacteria studied, any technique based on a Poisson distribution cannot be used throughout the entire growth period. That correction for population distribution is not necessary is encouraging, since it extends the range of types of micro-organisms which may be counted electronically.

\section{CONCLUSIONS}

It is concluded that it is possible to count growing bacterial populations electronically even though intra-species and inter-genus variations exist. These variations are the result of factors such as: the given organism, age, distribution and growth rate at the time of count, dilution and counting media, aperture diameter and magnitude of the current field in the electronic counting system.

This article contains information presented at the 1962 Annual Meeting of the American Society of Microbiology.

\section{REFERENCES}

Brecher, G., Jakobek, E. F., Schneideman, M. A., Williams, G. F. \& Schmitt, P. J. (1962). Size distribution of erythrocytes. Ann. N.Y. Acad. Sci. 99, Article 2, 242.

Dixon, W. J. \& Massey, F. J. (1957). Introduction to Statistical Analysis, 2nd ed., p. 328. New York: McGraw-Hill Book Company.

Swanton, E. M., Curby, W. A. \& Lind, H. E. (1962). Experiences with the Coulter counter in bacteriology. J. appl. Microbiol. 10, 480.

Toennies, G., Iszard, L. Rogers, N. B. \& Shockman, G. D. (1961). Cell multiplication studies with an electronic particle counter. J. Bact. 82, 857.

Truant, J. P., Brett, W. A. \& Merckel, K. E. (1962). Application of an electronic counter in the evaluation of significant bacteriuria. Henry Ford Hosp. Med. Bull. 10, 359. 